\title{
Reduction of a Mixed Characteristic Problem to the Initial Cauchy Problem for Nonlinear Oscillation Equations
}

\author{
Rusudan Bitsadze ${ }^{1, *}$ \\ ${ }^{1}$ Department of Mathematics, Georgian Technical University, Tbilisi, Georgia \\ *Corresponding author: bitsadze.r@gmail.com
}

\begin{abstract}
For the well-known nonlinear oscillation equation, we consider a nonlinear mixed characteristic problem, which is a nonlinear analogue of the Darboux problem and consists in the simultaneous definition of a solution and its regular propagation domain. The question of solvability of the formulated problem is solved by the method of characteristics and reduction to the Cauchy problem.
\end{abstract}

Keywords: characteristics, general integral, definition domain, initial problem

\section{Introduction}

As is known, the carrier of the Darboux problem for linear equations consists of two curves drawn from the common point of these curves [1]. One of these curves is characteristic, and the other has no characteristic direction anywhere. 
The characteristics of linear hyperbolic equations are completely defined by means of principal coefficients. In nonlinear cases these coefficients already depend on the sought solution and its lower derivatives. Since the characteristics, too, depend on them, the linear formulation of a mixed characteristic problem cannot be automatically extended to the case of nonlinear equations which are of particular interest from the standpoint of application [2]. Therefore, the formulations of mixed problems for such equations should be revised taking into account general characteristic invariants [3-7].

In this paper, an attempt is made to formulate correctly a partially characteristic problem for a quasilinear equation that has numerous practical and theoretical applications $[8,9]$ and arises when studying nonlinear oscillations

$$
x^{2}\left(u_{y}^{4} u_{x x}-u_{y y}\right)=c u u_{y}^{4}, c=\text { const }
$$

Equation (1) is interesting by the degeneracy of its order and, perhaps, by the hyperbolicity, too. The former is completely defined and occurs on the coordinate axis. The parabolic degeneracy [10] depends on the behavior of the derivative $u_{y}$ of an unknown solution $u(x, y)$. Hence the set of points of this degeneracy is not a priori prescribed in this case and has to be defined simultaneously with a solution.

Since the set of points of parabolic degeneracy and the characteristics are not defined by the equation, they have to be somehow defined by the conditions of the problem. For this, we need all characteristic rules of equation (1).

\section{Basic Part}

The characteristic roots of equation (1)

$$
\lambda_{1}=u_{y}^{-2}, \quad \lambda_{2}=-u_{y}^{-2}
$$

give the differential relations of characteristic directions

$$
u_{y}^{2} d y-d x=0, \quad u_{y}^{2} d y+d x=0
$$

If, taking into account (2), we consider equation (1), we come to the differential characteristic relations

$$
x^{2} u_{y}^{4} d u_{x}-x^{2} u_{y}^{2} d u_{y}-c u_{y}^{4} u d x=0, \quad x^{2} u_{y}^{4} d u_{x}+x^{2} u_{y}^{2} d u_{y}-c u_{y}^{4} u d x=0
$$


The following theorem $[11,12]$ is true.

Theorem 1. Assuming $c>-\frac{1}{4}$, each of the characteristic systems of equation (1) admits exactly two first integrals and they are represented explicitly as

$$
\left\{\begin{array}{l}
\xi \equiv\left(u_{y}^{-1}+u_{x}\right) x^{\alpha}-\alpha u x^{\alpha-1} \\
\xi_{1} \equiv\left(u_{y}^{-1}+u_{x}\right) x^{1-\alpha}-(1-\alpha) u x^{-\alpha}
\end{array},\right.
$$

for the family of the root $\lambda_{1}$, and as

$$
\left\{\begin{array}{l}
\eta \equiv\left(u_{y}^{-1}-u_{x}\right) x^{\alpha}+\alpha u x^{\alpha-1} \\
\eta_{1} \equiv\left(u_{y}^{-1}-u_{x}\right) x^{1-\alpha}+(1-\alpha) u x^{-\alpha}
\end{array}, \quad \alpha=\frac{1}{2}(1+\sqrt{4 c+1})\right.
$$

for the family of the root $\lambda_{2}$.

By virtue of these two pairs of first integrals $\left(\xi, \xi_{1}\right)$ and $\left(\eta, \eta_{1}\right)$, which are actually characteristic invariants, it follows that in the class of hyperbolic solutions we can construct two intermediate integrals

$$
\xi_{1}=\varphi^{\prime}(\xi), \quad \eta_{1}=\varphi^{\prime}(\eta)
$$

of equation (1) [12]. In these integrals, $\varphi, \psi$ are arbitrary smooth functions such that they ensure the differentiability of the sought solution up to the second order.

Theorem 2. If $\varphi, \psi \in C^{3}\left(R^{\prime}\right)$, then equation (1) is equivalent to the triple of the following relations $[11,12]$

$$
\begin{aligned}
& x=\left(\frac{\varphi^{\prime}(\xi)+\psi^{\prime}(\eta)}{\xi+\eta}\right)^{\frac{1}{1-2 \alpha}}, \\
& y=\frac{1}{4(1-2 \alpha)}\left[(\xi+\eta)\left(\psi^{\prime}(\eta)-\varphi^{\prime}(\xi)\right)+2(\varphi(\xi)-\psi(\eta))\right] \\
& u=\frac{1}{1-2 \alpha}\left[\xi\left(\frac{\varphi^{\prime}(\xi)+\psi^{\prime}(\eta)}{\xi+\eta}\right)^{\frac{1-\alpha}{1-2 \alpha}}-\varphi^{\prime}(\xi)\left(\frac{\varphi^{\prime}(\xi)+\psi^{\prime}(\eta)}{\xi+\eta}\right)^{\frac{\alpha}{1-2 \alpha}}\right] .
\end{aligned}
$$


To relations (5)-(7) we come from equation (1) without any additional conditions. By removing arbitrary parameters $\varphi, \psi$ from these relations we return to equation (1). Hence this triple of relations can be taken as a general integral of equation (1), and the invariants $\xi$, $\eta$ as characteristic variables.

However the above-constructed general integral (5)-(7) does not define in any way at least one characteristic of either of the families in order to take it as a data carrier of a mixed characteristic problem. Hence we have to choose such a characteristic arbitrarily, at our discretion. Suppose it is some arc $\gamma$ of continuously differentiable curvature given in explicit form

$$
\gamma: y=g(x), \quad 0<a \leq x \leq b
$$

The function $g(x)$ is assumed to be strictly monotonic, and the arc $\gamma$ to be ascending. Without loss of generality it can be assumed that

$$
g(a)=0
$$

Let the function $h(x)$ given on some segment $[a, d]$ be twice continuously differentiable and compressing this segment by a segment $[a, b]$. It is assumed that $h(x)$ satisfies the conditions

$$
h(a)=a, \quad h(d)=b, \quad h^{\prime}<0
$$

Mixed characteristic problem. Find a regular hyperbolic solution $u(x, y)$ of equation (1) and simultaneously define its propagation domain if along this solution the curve $\gamma$ is characteristic, the solution itself satisfies the conditions

$$
u(a, 0)=\vartheta, \quad u_{x}(a, 0)=\theta
$$

and each pair of points $(x, 0),(h(x), g(h(x)))$ connected with the mapping of $h$ belongs to the respective general characteristic of the family of the root $\lambda_{2}$.

According to the formulation of the problem, the curve $\gamma$ is actually attributed to the family of characteristics of the root $\lambda_{1}$. This is equivalent to the equality

$$
g^{\prime}(x)=u_{y}^{-2}(x, g(x))
$$


Thus we can define two variants of values of the derivative $u_{y}$ along the curve $\gamma$ :

$$
u_{y}=\frac{1}{ \pm \sqrt{g^{\prime}(x)}}
$$

Of them we choose the arithmetic value of the root. The reasoning for the other root is analogous. To solve the problem, along with (10) we also need to define on the arc $\gamma$ the values of a solution $u$ and its derivative $u_{x}$. To this end, we have to use the characteristic invariants $\xi, \xi_{1}$ of the family $\lambda_{1}$. The values of $u$ and $u_{x}$ at the initial point $(a, 0)$ of the curve $\gamma$ are known. Using (8), (10), we calculate the characteristic invariants $\xi, \xi_{1}$ at the point $(a, 0)$, for which we introduce the notation

$$
\begin{gathered}
\left.\xi\right|_{(a, 0)}=\left(\sqrt{g^{\prime}(a)}+\theta\right) a^{\alpha}-\alpha \vartheta a^{\alpha-1} \equiv[\xi]_{a}, \\
\left.\xi_{1}\right|_{(a, 0)}=\left(\sqrt{g^{\prime}(a)}+\theta\right) a^{1-\alpha}-(1-\alpha) \vartheta a^{-\alpha} \equiv\left[\xi_{1}\right]_{a} .
\end{gathered}
$$

Since the characteristic invariants $\xi, \xi_{1}$ take constant values along $\gamma$, we have

$$
\begin{array}{r}
{\left.\left[\left(u_{y}^{-1}+u_{x}\right) x^{\alpha}-\alpha u x^{\alpha-1}\right]\right|_{\gamma}=[\xi]_{a},} \\
{\left.\left[\left(u_{y}^{-1}+u_{x}\right) x^{1-\alpha}-(1-\alpha) u x^{-\alpha}\right]\right|_{\gamma}=\left[\xi_{1}\right]_{a} .}
\end{array}
$$

Treating these two relations as a system with respect to $u$ and $u_{x}$, we define their values on $\gamma$.

Thus, we have managed to define the values of the sought solution and its first order derivatives along the characteristic $\gamma$. These values should be used to define them outside the characteristic and to establish the boundaries of their propagation. This can be done in several ways. One of them is to reduce problem (1), (8) to the initial Cauchy problem with data on the segment

$$
\Delta=\{(x, y) \mid a \leq x \leq d, y=0\}
$$

To define $u(x, 0), u_{x}(x, 0)$ and $u_{y}(x, 0)$ on $[a, d]$, let us draw from an arbitrary point $P(x, 0), a<x \leq d$, the characteristic $\Gamma$ of the family of the root $\lambda_{2}$, which, by the conditions 
of problem (1), (8), intersects the characteristic $r$ at the point $M(h(x), g(h(x)))$. The invariants $\eta$ and $\eta_{1}$ are assumed to be constant along the characteristic $\Gamma$.

Since the values of $\eta, \eta_{1}$ at the point $M$

$$
\begin{gathered}
\left.\eta\right|_{M}=2 \sqrt{g^{\prime}(h(x))} h^{\alpha}(x)-[\xi]_{a}, \\
\left.\eta_{1}\right|_{M}=2 \sqrt{g^{\prime}(h(x))} h^{1-\alpha}(x)-\left[\xi_{1}\right]_{a},
\end{gathered}
$$

remain unchanged along the $\operatorname{arc} \Gamma$, including the point $(x, 0)$ too, the following equalities will be fulfilled

$$
\eta(x, 0)=\left.\eta\right|_{M}, \quad \eta_{1}(x, 0)=\left.\eta_{1}\right|_{M} .
$$

Writing these invariants in explicit form, we obtain

$$
\begin{aligned}
& {[\eta]_{x} \equiv\left(u_{y}^{-1}(x, 0)-u_{x}(x, 0)\right) x^{\alpha}+\alpha u(x, 0) x^{\alpha-1}=2 \sqrt{g^{\prime}(h(x))} h^{\alpha}(x)-[\xi]_{i},} \\
& {\left[\eta_{1}\right]_{x} \equiv\left(u_{y}^{-1}(x, 0)-u_{x}(x, 0)\right) x^{1-\alpha}+(1-\alpha) u(x, 0) x^{-\alpha}=2 \sqrt{g^{\prime}(h(x))} h^{1-\alpha}(x)-\left[\xi_{1}\right]_{u} .}
\end{aligned}
$$

Let us treat these equalities as a linear algebraic system and define the sought solution at an arbitrary point $(x, 0)$ of the segment $\Delta$

$$
\begin{array}{r}
u(x, 0)=\frac{2}{2 \alpha-1} \sqrt{g^{\prime}(h(x))}\left(h^{\alpha}(x) x^{1-\alpha}-h^{1-\alpha}(x) x^{\alpha}\right)-\frac{1}{2 \alpha-1}[\xi]_{a} x^{1-\alpha} \\
+\frac{1}{2 \alpha-1}\left[\xi_{1}\right]_{a} x^{\alpha} \equiv \tau(x) .
\end{array}
$$

For this it suffices to define at the same points the first order derivatives $u_{x}(x, 0), u_{y}(x, 0)$ of the sought solution $u(x, y)$. The first of these derivatives is obtained by direct differentiation of (14)

$$
\begin{aligned}
u_{x}(x, 0) & =\frac{1}{2 \alpha-1} \frac{g^{\prime \prime}(h(x)) \cdot h^{\prime}(x)}{\sqrt{g^{\prime}(h(x))}}\left(h^{\alpha}(x) x^{1-\alpha}-h^{1-\alpha}(x) x^{\alpha}\right)+ \\
+\frac{2}{2 \alpha-1} & \sqrt{g^{\prime}(h(x))}\left(\alpha h^{\alpha-1}(x) h^{\prime}(x) x^{1-\alpha}\right. \\
& \left.+(1-\alpha) h^{\alpha}(x) x^{-\alpha}-(1-\alpha) h^{-\alpha}(x) h^{\prime}(x) x^{\alpha}-\alpha h^{1-\alpha}(x) x^{\alpha-1}\right)
\end{aligned}
$$




$$
-\frac{1-\alpha}{2 \alpha-1}[\xi]_{a} x^{-\alpha}+\frac{\alpha}{2 \alpha-1}\left[\xi_{1}\right]_{a} x^{\alpha-1}
$$

The other derivative $u_{y}$ is defined by substituting (14), (15) into (12) or (13)

$$
\begin{aligned}
& u_{y}(x, 0)=\left\{\frac { 2 \sqrt { g ^ { \prime } ( h ( x ) ) } } { 2 \alpha - 1 } \left[(\alpha-1) h^{\alpha}(x) x^{-\alpha}+(1-\alpha) h^{1-\alpha}(x) x^{\alpha-1}\right.\right. \\
& \left.+\alpha h^{\alpha-1}(x) h^{\prime}(x) x^{1-\alpha}-(1-\alpha) h^{-\alpha}(x) h^{\prime}(x) x^{\alpha}\right] \\
& \left.+\frac{1}{2 \alpha-1} \frac{g^{\prime \prime}(h(x)) \cdot h^{\prime}(x)}{\sqrt{g^{\prime}(h(x))}}\left(h^{\alpha}(x) x^{1-\alpha}-h^{1-\alpha}(x) x^{\alpha}\right)+\frac{1-\alpha}{2 \alpha-1}[\xi]_{a} x^{\alpha}\right\}^{-1} \equiv v(x) .
\end{aligned}
$$

So, we now have at our disposal all the data required for stating the initial problem which is equivalent to the mixed characteristic problem (1), (8).

We are to define a hyperbolic solution $u(x, y)$ of equation (1) by the initial disturbances

$$
\left.u\right|_{y=0}=\tau(x),\left.\quad u_{y}\right|_{y=0}=v(x), \quad a \leq x \leq d .
$$

where $\tau, v$ are given by (14), (16).

Because of the nonlinearity of equation (1) and depending on the initial disturbances $\tau, v$, the initial data carrier $\Delta$ may turn out to be the characteristic of either of the families. This is the cause for which the considered problem can be ill-posed or even unsolvable.

In order to avoid the transformation of the initial data carrier to the characteristic, we should find the conditions ensuring the a priori estimate

$$
0<\left|u_{y}(x, 0)\right|<\infty
$$

It is understood that these conditions should be expressed in terms of the parameters $g, h$ of problem (1), (17).

The above estimate excludes, for $y=0$, not only the characteristic direction of the carrier, but also the parabolic degeneracy of equation (1).

The assumptions $h \in C^{1}(\bar{J}), g \in C^{2}(\bar{I}), J \equiv(a, d), I \equiv(a, b)$ ensure the fulfillment of the condition

$$
u_{y}(x, 0) \neq 0, \quad x \in \bar{J}
$$


and the existence of minimal and maximal values of the functions $g^{\prime}(x),\left|g^{\prime \prime}(x)\right|$ on $\bar{I}$ and of $|h(x)|$ on $\bar{J}$. We denote by $n$ the smallest of minimal values and by $N$ the largest of maximal values. We obtain the estimate

$$
\left|u_{y}(x, 0)\right|<+\infty, \quad x \in \bar{J}
$$

if one of the following conditions is fulfilled:

$$
\begin{aligned}
2 \sqrt{N}\left[(\alpha-1)\left(\frac{a}{d}\right)^{\alpha}-(\alpha-1)\left(\frac{d}{a}\right)^{\alpha-1}\right. & \left.-\alpha\left(\frac{b}{a}\right)^{\alpha-1} N-(\alpha-1)\left(\frac{d}{a}\right)^{\alpha} N\right] \\
& -\frac{N^{2}}{\sqrt{n}}\left(d^{\alpha} a^{1-\alpha}-d^{1-\alpha} a^{\alpha}\right)-(\alpha-1)[\xi]_{a} a^{\alpha}>0,
\end{aligned}
$$

where the value $[\xi]_{a}$ is given by formula (11), and $\alpha>1$,

$$
\begin{aligned}
\sqrt{N} \eta_{*}\left(1+\operatorname{sig} \eta_{*}\right)+\sqrt{n} \eta_{*}\left(1-\operatorname{sig} \eta_{*}\right) & +\frac{N^{2}}{\sqrt{n}}\left(d^{\alpha} a^{1-\alpha}-d^{1-\alpha} a^{\alpha}\right) \\
& -(\alpha-1)[\xi]_{a}\left(a^{\alpha} \frac{1+\operatorname{sig}[\xi]_{a}}{2}+d^{\alpha} \frac{1-\operatorname{sig}[\xi]_{a}}{2}\right)<0,
\end{aligned}
$$

where

$$
\begin{gathered}
\eta_{*} \equiv(\alpha-1)\left(\frac{b}{a}\right)^{\alpha}-(\alpha-1)\left(\frac{a}{b}\right)^{\alpha-1}-\alpha\left(\frac{a}{d}\right)^{\alpha-1} n-(\alpha-1)\left(\frac{a}{b}\right)^{\alpha} n, \quad \alpha>1, \\
g^{\prime \prime}(x) \leq 0, \quad x \in \bar{I}, \quad \alpha=1, \\
\sqrt{N} \eta^{*}\left(1-\operatorname{sig} \eta^{*}\right)+\sqrt{n} \eta^{*}\left(1+\operatorname{sig} \eta^{*}\right)-\frac{N^{2}}{\sqrt{n}}(d-a) \\
+(1-\alpha)[\xi]_{a}\left[a^{\alpha} \frac{1+\operatorname{sig}[\xi]_{a}}{2}+d^{\alpha} \frac{1-\operatorname{sig}[\xi]_{a}}{2}\right]>0,
\end{gathered}
$$

where

$$
\eta^{*} \equiv(\alpha-1)\left(\frac{b}{a}\right)^{\alpha}+(1-\alpha)\left(\frac{a}{d}\right)^{1-\alpha}-\alpha\left(\frac{d}{a}\right)^{1-\alpha} N+(1-\alpha)\left(\frac{a}{d}\right)^{\alpha} n, \quad \frac{1}{2}<\alpha<1,
$$




$$
\begin{aligned}
\eta_{0} \sqrt{n}\left(1-\operatorname{sig} \eta_{0}\right)+\eta_{0} \sqrt{N}\left(1+\operatorname{sig} \eta_{0}\right) & +\frac{n^{2}}{\sqrt{n}}(d-a) \\
& +(1-\alpha)[\xi]_{a}\left(d^{\alpha} \frac{1+\operatorname{sig}[\xi]_{a}}{2}+a^{\alpha} \frac{1-\operatorname{sig}[\xi]_{a}}{2}\right)<0,
\end{aligned}
$$

where

$$
\eta_{0} \equiv(\alpha-1)\left(\frac{a}{d}\right)^{\alpha}+(1-\alpha)\left(\frac{b}{a}\right)^{1-\alpha}-\alpha\left(\frac{a}{b}\right)^{1-\alpha} n+(1-\alpha)\left(\frac{d}{a}\right)^{\alpha} N, \quad \frac{1}{2}<\alpha<1 .
$$

Thus, if one of conditions (18)-(23) is fulfilled, then neither of the characteristic invariants (3), (4) on the initial data carrier $\Delta$ is constant. Hence the derivatives of traces of the invariants $\xi, \eta$ on the segment $\Delta$ are different from zero. Denoting the values of these invariants on $\Delta$ by $M(x)$ and $\Lambda(x)$, respectively, we have

$$
\begin{gathered}
M^{\prime}(x)=\left[\left(v^{-1}(x)+\tau^{\prime}(x)\right) x^{\alpha}-\alpha \tau(x) x^{\alpha-1}\right]^{\prime} \neq 0, \\
\Lambda^{\prime}(x)=\left[\left(v^{-1}(x)-\tau^{\prime}(x)\right) x^{\alpha}+\alpha \tau(x) x^{\alpha-1}\right]^{\prime} \neq 0 .
\end{gathered}
$$

Let us now write the conditions of problem (1), (17) in terms of the characteristic variables $\xi, \eta$.

On the plane corresponding to the variables $\xi, \eta$, the initial data carrier $\Delta$ is represented by the parametric equations

$$
\Delta_{c}: \xi=M(x), \quad \eta=\Lambda(x), \quad x \in[a, d] .
$$

The mapping of the segment $\Delta$ on the characteristic plane can be represented by the interdependence of $\xi$ and $\eta$ by means of the functions $\Lambda, M$ provided that they are invertible. Assuming that these functions exist, we denote them by $\mu, \lambda$, respectively. The first of them will be defined on the closed interval $\Delta_{1} \equiv[M(a), M(d)]$, and the other - on $\Delta_{2} \equiv[\Lambda(a), \Lambda(d)]$, where the curve $\Delta_{c}$ is represented by the equation

$$
\eta=\Lambda(\mu(\xi)), \quad \xi \in \Delta_{1}
$$

On the other hand, the argument $x$ on the characteristic plane is defined as a function of the variables $\xi, \eta$ by formula (5). On the curve $\Delta_{c}$, this argument can be represented by a 
function of the argument $\xi$. This is done by replacing the values $\eta$ with the expression $\Lambda(\mu(\xi))$ in $(5)$

$$
x(\xi, \Lambda(\mu(\xi)))=\mu(\xi) .
$$

From (24), (25) we obtain

$$
\frac{\varphi^{\prime}(\xi)+\psi^{\prime}[\Lambda(\mu(\xi))]}{\xi+\Lambda(\mu(\xi))}=\mu^{1-2 \alpha}(\xi), \quad \xi \in \Delta_{1},
$$

whence we can define the unknown functions $\varphi, \psi$. To this end, we observe that the equality

$$
\psi^{\prime}[\Lambda(\mu(\xi))]=\xi \mu^{1-2 \alpha}(\xi)-\varphi^{\prime}(\xi)+\Lambda(\mu(\xi)) \mu^{1-2 \alpha}(\xi), \quad \xi \in \Delta_{1}
$$

is fulfilled along the curve $\Delta_{c}$.

The first of conditions (17) written in terms of characteristic variables takes the form

$$
u(\xi, \Lambda(\mu(\xi)))=\tau(\mu(\xi))
$$

Combining now equality (28) with representation (7) of the value $u(\xi, \eta)$, we come to a relation which, taking into account the expression of $\mu(\xi)$, can be written in the form

$$
\frac{1}{1-2 \alpha}\left(\xi \mu^{1-\alpha}(\xi)-\varphi^{\prime}(\xi) \mu^{\alpha}(\xi)\right)=\tau(\mu(\xi))
$$

This relation makes it possible to define the derivative $\varphi^{\prime}$ of the sought function $\varphi(\xi)$ :

$$
\varphi^{\prime}(\xi)=\xi \mu^{1-2 \alpha}(\xi)-(1-2 \alpha) \tau(\mu(\xi)) \mu^{-\alpha}(\xi)
$$

The substitution of the obtained expression into (46) yields the equality

$$
\psi^{\prime}[\Lambda(\mu(\xi))]=\mu^{1-2 \alpha}(\xi) \Lambda(\mu(\xi))+(1-2 \alpha) \tau(\mu(\xi)) \mu^{-\alpha}(\xi)
$$

which, after replacing the argument $\Lambda(\mu(\xi))$ with the new argument $\eta$, can be rewritten as

$$
\psi^{\prime}(\eta)=\lambda^{1-2 \alpha}(\eta) \cdot \eta+(1-2 \alpha) \tau(\lambda(\eta)) \lambda^{-\alpha}(\eta)
$$

By integrating (29), (30), for arbitrary functions $\varphi(\xi)$ and $\psi(\eta)$ figuring in the general integral (5)-(7) of equation (1), we have 


$$
\begin{aligned}
& \varphi(\xi)=\int_{\xi^{0}}^{\xi} t \mu^{1-2 \alpha}(t) d t-(1-2 \alpha) \int_{\xi^{0}}^{\xi} \tau(\mu(t)) \mu^{-\alpha}(t) d t+\varphi\left(\xi^{0}\right), \quad \xi^{0} \equiv M\left(x^{0}\right) \\
& x^{0} \in[a, d], \quad \xi \in \Delta_{1}, \\
& \psi(\eta)=\int_{\eta^{0}}^{\eta} t \lambda^{1-2 \alpha}(t) d t-(1-2 \alpha) \int_{\eta^{0}}^{\eta} \tau(\lambda(t)) \lambda^{-\alpha}(t) d t+\psi\left(\eta^{0}\right), \quad \eta^{0} \equiv \Lambda\left(x^{0}\right), \\
& x^{0} \in[a, d], \quad \eta \in \Delta_{2} .
\end{aligned}
$$

Hence, taking (6) into account, we obtain

$$
\begin{gathered}
y=\frac{1}{4(1-2 \alpha)}\left\{( \xi + \eta ) \left[\eta \lambda^{1-2 \alpha}(\eta)+(1-2 \alpha) \tau(\lambda(\eta)) \lambda^{-\alpha}(\eta)\right.\right. \\
\left.-\xi \mu^{1-2 \alpha}(\xi)+(1-2 \alpha) \tau(\mu(\xi)) \mu^{-\alpha}(\xi)\right] \\
+2 \int_{\xi^{0}}^{\xi} t \mu^{1-2 \alpha}(t) d t-2(1-2 \alpha) \int_{\xi^{0}}^{\xi} \tau(\mu(t)) \mu^{-\alpha}(t) d t+2 \varphi\left(\xi^{0}\right) \\
\left.-2 \int_{\eta^{0}}^{\eta} t \lambda^{1-2 \alpha}(t) d t-2(1-2 \alpha) \int_{\eta^{0}}^{\eta} \tau(\lambda(t)) \lambda^{-\alpha}(t) d t-2 \psi\left(\eta^{0}\right)\right\},
\end{gathered}
$$

where $\xi \in \Delta_{1}, \eta \in \Delta_{2}, x^{0} \in[a, d]$.

To define the constant value

$$
c^{0} \equiv 2\left[\varphi\left(\xi^{0}\right)-\psi\left(\lambda\left(\eta^{0}\right)\right)\right]
$$

we use the fact that the variable $y$ takes zero values at all points of the curve $\Delta_{c}$. In particular $y$ also vanishes for $\xi=\xi^{0}=M\left(x^{0}\right), \eta=\eta^{0}=\Lambda\left(x^{0}\right)$

$$
y\left(\xi^{0}, \eta^{0}\right)=0
$$

where $x^{0} \in[a, d]$ is the fixed value of the argument. Hence, by (33), we obtain the value $c^{0}$ and, using relations (5)-(7), (27), (29) and substituting the value $c^{0}$ into (33), we finally construct the integral of problem (1), (17) in terms of characteristic variables in the rectangle $\Delta_{1} \times \Delta_{2}:$ 


$$
\begin{aligned}
& x=F(\xi, \eta) \equiv(\xi+\eta)^{\frac{1}{2 \alpha-1}}\left[\xi \mu^{1-2 \alpha}(\xi)-(1-2 \alpha) \tau(\mu(\xi)) \mu^{-\alpha}(\xi)\right. \\
& \left.+\eta \lambda^{1-2 \alpha}(\eta)+(1-2 \alpha) \tau(\lambda(\eta)) \lambda^{-\alpha}(\eta)\right]^{\frac{1}{1-2 \alpha}} \\
& y=G(\xi, \eta) \equiv \frac{1}{4(1-2 \alpha)}\left\{( \xi + \eta ) \left[\eta \lambda^{1-2 \alpha}(\eta)+(1-2 \alpha) \tau(\lambda(\eta)) \lambda^{-\alpha}(\eta)\right.\right. \\
& \left.-\xi \mu^{1-2 \alpha}(\xi)+(1-2 \alpha) \tau(\mu(\xi)) \mu^{-\alpha}(\xi)\right] \\
& +2 \int_{\xi^{0}}^{\xi} t \mu^{1-2 \alpha}(t) d t-2(1-2 \alpha) \int_{\xi^{0}}^{\xi} \tau(\mu(t)) \mu^{-\alpha}(t) d t \\
& -2 \int_{\eta^{0}}^{\eta} t \lambda^{1-2 \alpha}(t) d t-2(1-2 \alpha) \int_{\eta^{0}}^{\eta} \tau(\lambda(t)) \lambda^{-\alpha}(t) d t \\
& \left.+\left(\xi^{0}+\eta^{0}\right)\left[\left(\xi^{0}-\eta^{0}\right)\left(x^{0}\right)^{1-2 \alpha}-2(1-2 \alpha)\left(x^{0}\right)^{-\alpha} \tau\left(x^{0}\right)\right]\right\}, \\
& u=\frac{1}{4(1-2 \alpha)}\left\{\xi \left[( \xi + \eta ) ^ { - 1 } \left(\xi \mu^{1-2 \alpha}(\xi)-(1-2 \alpha) \tau(\mu(\xi)) \mu^{-\alpha}(\xi)\right.\right.\right. \\
& \left.\left.+\eta \lambda^{1-2 \alpha}(\eta)+(1-2 \alpha) \tau(\lambda(\eta)) \lambda^{-\alpha}(\eta)\right)\right]^{\frac{1-\alpha}{1-2 \alpha}} \\
& -\left[\xi \mu^{1-2 \alpha}(\xi)-(1-2 \alpha) \tau(\mu(\xi)) \mu^{-\alpha}(\xi)\right]\left[( \xi + \eta ) ^ { - 1 } \left(\xi \mu^{1-2 \alpha}(\xi)-(1-2 \alpha) \tau(\mu(\xi)) \mu^{-\alpha}\right.\right. \\
& \left.\left.\left.+\eta \lambda^{1-2 \alpha}(\eta)+(1-2 \alpha) \tau(\lambda(\eta)) \lambda^{-\alpha}(\eta)\right)\right]^{\frac{\alpha}{1-2 \alpha}}\right\},
\end{aligned}
$$

where $x^{0} \in[a, d]$ is arbitrary and $\xi \in \Delta_{1}, \eta \in \Delta_{2}$.

At first sight, the value $y$ depends on $x^{0}$. However this does not influence the final result. Indeed, assuming $y$ to be a function of three variables $\xi, \eta, x^{0}$ and differentiating it with respect to $x^{0}$, we come to the equality

$$
\frac{\partial y}{\partial x^{0}}=\frac{1}{4}\left(\xi^{0}+\eta^{0}\right)\left[\xi^{0}-\eta^{0}+2 \alpha\left(x^{0}\right)^{\alpha-1} \tau\left(x^{0}\right)-2\left(x^{0}\right)^{\alpha} \tau^{\prime}\left(x^{0}\right)\right]\left(x^{0}\right)^{-2 \alpha} \text {. }
$$

Using the expressions of $\xi^{0}$ and $\eta^{0}$, we conclude that

$$
\frac{\partial y}{\partial x^{0}} \equiv 0
$$


which immediately implies the validity of our proposition.

Therefore the choice of a point $x^{0} \in[a, d]$ does not actually influence the correct formulation of the Cauchy problem.

We have thereby finished the construction of the integral of problem (1), (17) and therefore of problem (1), (8), but we should also indicate the domain into which this integral propagates from the data carrier. The propagation domain is completely defined by relations (34), (35) which express $x, y$ as functions of the arguments $\xi, \eta$. Consequently, on the plane of variables $x, y$, the definition domain of the integral of problem (1), (8) will be the mapping of the characteristic rectangle $\Delta_{1} \times \Delta_{2}$. The boundary of this domain $D$ entirely consists of the characteristics drawn from the finite points of the carrier.

The case where on the characteristic $\gamma$ the derivative $u_{y}$ has the opposite sign is considered analogously.

In some cases, we manage to express a solution of the Cauchy problem in terms of the variables $x, y$. This result is achieved when conditions (34), (35) enable us to define the characteristic invariants $\xi, \eta$ in terms of the arguments $x, y$. Such situations can be obviously illustrated by concrete examples, one of which is given below.

\section{Examples}

Let us consider the case

$$
c=\frac{3}{4}, \quad \tau(x)=x, \quad v(x)=\frac{2}{3}, \quad x \in[a, d], \quad a>0 .
$$

The characteristic invariants are

$$
\begin{gathered}
\xi \equiv\left(u_{y}^{-1}+u_{x}\right) x^{\frac{3}{2}}-\frac{3}{2} u x^{\frac{1}{2}}, \quad \eta \equiv\left(u_{y}^{-1}-u_{x}\right) x^{\frac{3}{2}}+\frac{3}{2} u x^{\frac{1}{2}}, \\
\xi_{1} \equiv\left(u_{y}^{-1}+u_{x}\right) x^{-\frac{1}{2}}+\frac{1}{2} u x^{-\frac{3}{2}}, \quad \eta_{1} \equiv\left(u_{y}^{-1}-u_{x}\right) x^{-\frac{1}{2}}-\frac{1}{2} u x^{-\frac{3}{2}} .
\end{gathered}
$$

The general integral of equation (1) in terms of characteristic variables $\xi, \eta$ is represented by the relations 


$$
\begin{aligned}
& x=\left(\frac{\varphi^{\prime}(\xi)+\psi^{\prime}(\eta)}{\xi+\eta}\right)^{-\frac{1}{2}}, \\
& y=-\frac{1}{8}\left((\xi+\eta)\left(\psi^{\prime}(\eta)-\varphi^{\prime}(\xi)\right)-2(\varphi(\xi)-\psi(\eta))\right), \\
& u=-\frac{1}{2}\left(\xi\left(\frac{\varphi^{\prime}(\xi)+\psi^{\prime}(\eta)}{\xi+\eta}\right)^{\frac{1}{4}}-\varphi^{\prime}(\xi)\left(\frac{\varphi^{\prime}(\xi)+\psi^{\prime}(\eta)}{\xi+\eta}\right)^{-\frac{3}{4}}\right) .
\end{aligned}
$$

From conditions (38) and formulas (39) it follows that on the characteristic plane, to the carrier $\Delta$ there corresponds a straight line segment

$$
\gamma: \eta=2 \xi, \quad a^{\frac{3}{2}} \leq x \leq d^{\frac{3}{2}}
$$

which is immediately obtained from the relations

$$
\xi=x^{\frac{3}{2}}, \quad \eta=2 x^{\frac{3}{2}} .
$$

Therefore

$$
\begin{aligned}
& \left.x(\xi, \eta)\right|_{\eta=2 \xi}=\xi^{\frac{2}{3}} \\
& \left.u(\xi, \eta)\right|_{\eta=2 \xi}=\xi^{\frac{2}{3}} \\
& \left.y(\xi, \eta)\right|_{\eta=2 \xi}=0 .
\end{aligned}
$$

The last equality immediately implies

$$
\left.y(\xi, \eta)\right|_{(\xi, \eta)=\left(a^{\frac{3}{2}}, 2 a^{\frac{3}{2}}\right)}=0 .
$$

Using (43), (45) we have the equality

$$
-\xi^{\frac{2}{3}}+\xi \varphi^{\prime}(\xi)=2 \xi^{\frac{2}{3}} .
$$

Hence it follows that on $\gamma$

$$
\varphi(\xi)=\frac{9}{2} \xi^{\frac{2}{3}}-\frac{9}{2} a+\varphi(a)
$$


By (41), (44) and (47) we conclude that the equality $\psi^{\prime}(\eta)=0$ is fulfilled on the segment $\gamma$ and therefore

$$
\psi(\eta)=\text { const }
$$

By virtue of (46)-(48) we obtain

$$
\psi(\eta)=-4 \frac{1}{2} a+\varphi(a)
$$

The substitution of the values $\varphi(\xi), \psi(\eta)$ into (41)-(43) leads to the relations

$$
\left\{\begin{array}{l}
x=\frac{1}{\sqrt{3}}(\xi+\eta)^{\frac{1}{2}} \xi^{-\frac{1}{6}} \\
x=\frac{3}{8}(\xi+\eta) \xi^{-1}-1 \frac{1}{8} \xi^{\frac{2}{3}}
\end{array}\right.
$$

Relations (49) are regarded as a functional system with respect to $\xi, \eta$ which should be defined as functions of the variables $x, y$. For this, the last equation of system (49) is rewritten as follows:

$$
y=1 \frac{1}{8}\left(x^{2} \xi^{-\frac{2}{3}}-\xi^{\frac{2}{3}}\right)
$$

From this relation we obtain

$$
\xi=\frac{1}{27}\left(\sqrt{16 y^{2}+81 x^{2}}-4 y\right)^{\frac{3}{2}}
$$

Therefore by virtue of (49)

$$
\xi+\eta=x^{2}\left(\sqrt{16 y^{2}+81 x^{2}}-4 y\right)
$$

Using relations (43), (47), (48), (51), (52) we find the desired solution

$$
u(x, y)=-\frac{1}{54} x^{-\frac{1}{2}}\left(\sqrt{16 y^{2}+81 x^{2}}-4 y\right)^{\frac{3}{2}}+\frac{9}{2} x^{\frac{3}{2}}\left(\sqrt{16 y^{2}+81 x^{2}}-4 y\right)^{-\frac{1}{2}} .
$$

The boundary of the definition domain of this equation consists of the following characteristics

$$
\xi(x, y)=a^{\frac{3}{2}}, \quad \eta(x, y)=2 d^{\frac{3}{2}}, \quad \xi(x, y)=d^{\frac{3}{2}}, \quad \eta(x, y)=2 a^{\frac{3}{2}}
$$


Explicit equations of these characteristics are immediately obtained by substituting the values $\sqrt{a^{3}}$ and $\sqrt{d^{3}}$ of the function $\xi$ into (50), and the values $2 \sqrt{d^{3}}$ and $2 \sqrt{a^{3}}$ of the function $\eta$ into formulas (51), (52)

$$
\begin{aligned}
& y=\frac{9}{8 a} x^{2}-\frac{9 a}{8}, \quad 3 x^{2}-\frac{1}{81}\left(\sqrt{16 y^{2}+81 x^{2}}-4 y\right)^{2}-\frac{2}{3} b^{\frac{3}{2}}\left(\sqrt{16 y^{2}+81 x^{2}}-4 y\right)^{\frac{1}{2}}=0, \\
& y=\frac{9}{8 d} x^{2}-\frac{9 d}{8}, \quad 3 x^{2}-\frac{1}{81}\left(\sqrt{16 y^{2}+81 x^{2}}-4 y\right)^{2}-\frac{2}{3} a^{\frac{3}{2}}\left(\sqrt{16 y^{2}+81 x^{2}}-4 y\right)^{\frac{1}{2}}=0 .
\end{aligned}
$$

\section{References}

[1] Goursat, E., Lecons sur l'intégration des équations aux dérivées partielles du second ordre à deux variables indépendantes. Tome II: La méthode de Laplace. Les systèmes en involutions. La methode de M. Darboux. Les équations de la première classe. Transformations des équations du second ordre. Généralisations diverses, 2 Notes A. Hermann, Paris, 1898.

[2] Bers, L., Mathematical Aspects of Subsonic and Transonic Gas Dynamics, Surveys in Applied Mathematics, vol. 3 John Wiley \& Sons, Inc., New York; Chapman \& Hall, Ltd., London, 1958.

[3] Gvazava, J., "On one nonlinear version of the characteristic problem with a free support of data”, Proc. A. Razmadze Math. Inst., 140. 91-107. 2006.

[4] Gvazava, J., "The mean value property for nonstrictly hyperbolic second order quasilinear equations and the nonlocal problems”, Proc. A. Razmadze Math. Inst. 135. 79-96. 2004.

[5] Klebanskaya, M., "Some nonlinear versions of Darboux and Goursat problems for a hyperbolic equation with parabolic degeneracy", in International Symposium on Differential Equations and Mathematical Physics dedicated to the 90th birthday anniversary of Academician I. Vekua, Tbilisi, Georgia, June 21-25. 1997.

[6] Gvazava, J.K., "Second-order nonlinear equations with complete characteristic systems and characteristic problems for them”. (Russian) Trudy Tbiliss. Mat. Inst. Razmadze Akad. Nauk Gruzin. SSR 87. 45-53. 1987. 
[7] Bitsadze, R., "On one version of the initial-characteristic Darboux problem for one equation of nonlinear oscillation", Reports of an Enlarged Session of the Seminar of I. Vekua Institute of Applied Mathematics 8 (1). 4-6. 1993.

[8] Kumei S. and Bluman G.W., "When nonlinear differential equations are equivalent to linear differential equations", SIAM J. Appl. Math. 42(5). 1157-1173. 1982.

[9] Olver P., Applications of Lie Groups to Differential Equations, Mir, Moscow, 1989.

[10] Tricomi F., Lectures on Partial Equations, Moscow, 1957.

[11] Bitsadze R.G., "General representation of solutions of a quasilinear equation of a nonlinear oscillations problem”, Soobshch. Akad. Nauk Gruzin. SSR 128 (3). 493-496. 1988 (in Russian).

[12] Bitsadze R., "Mixed characteristic problem for a nonlinear oscillation equation", (Russian) Sovrem. Mat. Prilozh. No. 89 (2013); translation in J. Math. Sci. (N.Y.) 206 (4). 329-340. 2015.

[13] Goursat E., Course of Mathematical Analysis, vol. 3, part I, Moscow, 1933. 